\title{
Multilevel Coding for Channels with Non-uniform Inputs and Rateless Transmission over the BSC
}

\author{
Jing Jiang and Krishna R. Narayanan \\ Department of Electrical and Computer Engineering, \\ Texas A\&M University, \\ College Station, TX, 77840, U.S.A
}

\begin{abstract}
We consider coding schemes for channels with nonuniform inputs (NUI), where standard linear block codes can not be applied directly. We show that multilevel coding (MLC) with a set of linear codes and a deterministic mapper can achieve the information rate of the channel with NUI. The mapper, however, does not have to be one-to-one. As an application of the proposed MLC scheme, we present a rateless transmission scheme over the binary symmetric channel (BSC).
\end{abstract}

\section{INTRODUCTION}

Consider a discrete memoryless channel (DMC) with input $X \in \mathcal{X}$, output $Y \in \mathcal{Y}$ and let $P_{X}$ be the input distribution. When $P_{X}$ is the uniform distribution on $\mathcal{X}$, denoted by unif $(\mathcal{X})$, it is well known that linear codes can be directly used to achieve the information rate corresponding to $P_{X}$ [1]. When $|\mathcal{X}|=2^{m}$, binary linear codes along with multilevel coding (MLC) suffice to achieve the information rate corresponding to unif $(\mathcal{X})$. Given extensive recent results on designing good LDPC codes for binary input symmetric channels [2], [3], it suffices to say that LDPC codes provide a good practical solution to this communication problem.

However, in many cases, we are interested in input distributions which are not the uniform distribution. This could be because the capacity achieving distribution is non-uniform (for example, the Z channel [4]). Or, other signalling constraints may force us to use non-uniform inputs. An example of this is optical channels with cross-talks, where the probability of $1 \mathrm{~s}$ transmitted by each user $p_{1}=P(X=1)$ has to be constrained to be $p_{1} \ll 1 / 2$ to control the interference to other users [5]. In such scenarios, binary linear codes can not be applied directly since they can only induce the uniform distribution. We refer to such channels as 'channels with non-uniform inputs (NUI)'. The coding problem for such channels remains open [4].

This problem was previously studied by Ratzer and Mackay [5] [6]. In [6], they focused on designing inverse Huffmann code type mappers to induce the desired distribution. However, soft output decoding of the Huffman code is usually computationally complex and, further, the variable length nature of the mapping may incur catastrophic decoding errors. Alternatively, in [5], LDPC codes over GF(q) with deterministic mappers were used to induce the desired non-uniform distribution. The main drawback of this scheme is that the decoding complexity for the nonbinary LDPC code is significantly larger and the code optimization is very complicated.
In this paper, we first show that MLC using a set of binary linear codes and a deterministic mapper suffices to achieve the information rate of the channel with NUI. The mapper, however, does not necessarily have to be one-to-one. This scheme, discussed in Section 1 is shown to be optimal when the channel law is known at the transmitter. Although an MLC scheme with binary inputs can only induce dyadic input distributions, it is shown that via proper time sharing, the proposed MLC with a small number of layers can get close to the channel information rate for an arbitrary $P_{X}$. Compared with the previous works, the proposed MLC scheme not only has low complexity, but is theoretically justifiable as well.

As an important application of coding for channels with NUI, we consider the problem of rateless transmission over the binary symmetric channel (BSC). In [7], a simple layering, dithering (or interleaving) and repeating based rateless scheme was proposed for AWGN channel. In this paper, we extend (non-trivially) their results to the BSC case. Thanks to the degraded nature of the BSC, a similar layering scheme can be applied without a rate loss. However, in order to perform layering, the number of $1 \mathrm{~s}$ of the coded bits in each layer must be constrained. This is precisely where the proposed MLC scheme can be applied. We further show that repeating does not incur a rate loss in the low rate region over the BSC even for non-uniform inputs. Therefore, rateless transmission over the BSC becomes possible by simply layering, interleaving and repeating the proposed MLC block.

\section{CODING FOR CHANNELS With NON-UNIFORM INPUTS}

The problem of coding for channels with NUI can be dated back to Gallager. In [8], Gallager showed that binary linear codes can achieve the capacity of any DMC:

Theorem 1 Binary linear codes can be used to achieve the capacity of an arbitrary discrete memoryless channel.

We refer interested readers to [8] for the detailed proof. The main result of this theorem says that for any DMC, capacity can be achieved by a set of linear codes with a deterministic mapper under maximum likelihood decoding (MLD). However, as suggested by Gallager, finding practical decoding algorithms is a nontrivial problem. Note that in Gallager's proof, the key component, a deterministic mapper is used to induce the desired channel input distribution to achieve 


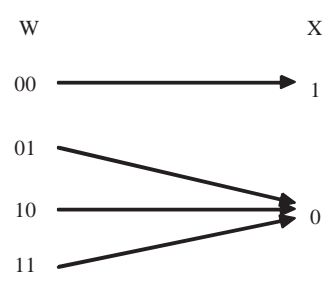

Fig. 1. An example of a deterministic mapper

the capacity of the DMC. The deterministic mapper can be defined as follows:

Definition 1 A deterministic mapping is a function $f: W \rightarrow$ $X$, where $W \in\{0,1\}^{m}$ and $X \in \mathcal{X}(\mathcal{X}$ is the set of all possible channel input symbols).

For example, consider the channel with NUI, i.e., $p_{1}=$ $P(X=1)=1 / 4$ and $p_{0}=P(X=0)=3 / 4$. A possible deterministic mapper is shown in Figure 11 where $W \in\{0,1\}^{2}$ and $X \in\{0,1\}$. Since $W$ is uniformly distributed, the mapper can induce the desired distribution on $\mathcal{X}$. Note that using linear codes with a deterministic mapper, we can only obtain probabilities of the form $k / 2^{m}$. However, by increasing $m$, we can approximate the desired distribution arbitrarily well.

Proposed Scheme: We first propose an MLC scheme to achieve the information rate of channels with NUI. The diagram of the proposed MLC scheme is shown in Figure 2 and the details of the scheme is as follows: Encoding: In each layer, $W_{i}$ is encoded using a capacity achieving binary linear code. The code rate of the $i^{\text {th }}$ layer is selected to be $R_{i}=I\left(W_{i} ; Y \mid W_{1}, \cdots, W_{i-1}\right)$. Then we induce the desired distribution on $\mathcal{X}$ from $W=\left[W_{1}, \cdots, W_{m}\right]$ using a deterministic mapper as suggested in Theorem 11 Decoding: At the decoder, we apply MSD: $W_{1}$ is first decoded and then $W_{2}$ is decoded based on $Y$ and the decision of $W_{1}$ and so forth until $W_{m}$ is decoded based on $Y$ and all the decisions from $W_{1}$ to $W_{m-1}$.

Now, we show that the information rate of a channel with NUI can be achieved by the above MLC scheme.

Theorem 2 The proposed coding scheme can achieve the information rate of the DMC with NUI, i.e., $\sum_{i=1}^{m} I\left(W_{i} ; Y \mid W_{1}, \cdots, W_{i-1}\right)=I(X ; Y)$.

Proof: We first show that the deterministic mapping from $W$ to $X$ does not incur a rate loss. Note that $W \rightarrow X \rightarrow Y$ forms a Markov chain. Expanding $I(W ; Y, X)$ in two ways, we have:

$$
\begin{aligned}
I(W ; Y, X) & =I(W ; X)+I(W ; Y \mid X) \\
& =I(W ; Y)+I(W ; X \mid Y)
\end{aligned}
$$

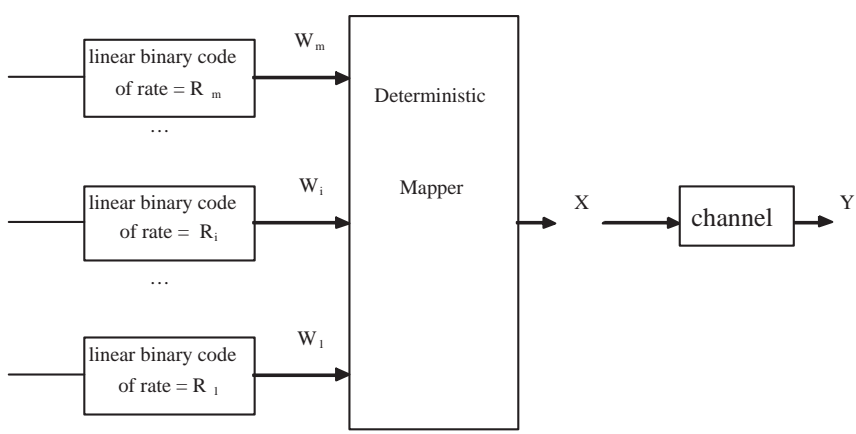

Fig. 2. System Model for the Proposed Coding Scheme for Constrained Input Channels

Due to the Markovian structure $I(W ; Y \mid X)=0$, thus, the mutual information between $W$ and $Y$ can be written as:

$$
\begin{aligned}
I(W ; Y) & =I(W ; X)-I(W ; X \mid Y) \\
& =(H(X)-H(X \mid W))-(H(X \mid Y)-H(X \mid W, Y)) \\
& =H(X)-H(X \mid Y)=I(X ; Y)
\end{aligned}
$$

(4) to (5) follows by the fact that $X$ is a function of $W$.

Hence, we can achieve the information rate using the proposed MLC. Since $W=\left[W_{1}, W_{2}, \cdots, W_{m}\right]$, the mapping from $W_{1}, W_{2}, \cdots, W_{m}$ to $W$ is a bijection. According to the chain rule of mutual information, we have:

$$
I(X ; Y)=I(W ; Y)=\sum_{i=1}^{m} I\left(W_{i} ; Y \mid W_{1}, \cdots, W_{i-1}\right)
$$

The proof generalizes the MLC proof in [9], where the mapping from $W$ to $X$ is a bijection. However, here, $W$ does not need to be a deterministic function of $X$, which suggests that the one-to-one type mappers (e.g., inverse Huffman code [6]) are not required in order to achieve the information rate. Essentially, what is needed is a deterministic mapper, which shapes the uniform distribution obtained from the coded bits of the linear codes to be the desired channel NUI distribution. Besides, the theorem implies that in each layer, standard binary linear codes, such as binary LDPC codes suffice.

Example 1: We give an example of the proposed MLC scheme over a BSC with NUI. In Figure 3 the information rate is plotted as a function of the channel crossover probability $h$. The probability of $1 \mathrm{~s}$ at the channel input is fixed to be $p_{1}=1 / 4$. We can see that the proposed MLC can achieve the information rate supported by the channel. In contrast, time sharing a linear code with 0 s will incur a significant rate loss. Besides, note that the mapper will introduce memory across the layers. Therefore, bit-interleaved-coded-modulation (BICM) without iterative demodulation (the demapper generates bit-level soft information for each layer by ignoring the correlation across the layers) also incurs a significant rate loss. For $\mathrm{Z}$ channels, similar phenomenon is observed. Due to the page limit, the results are not shown here. 


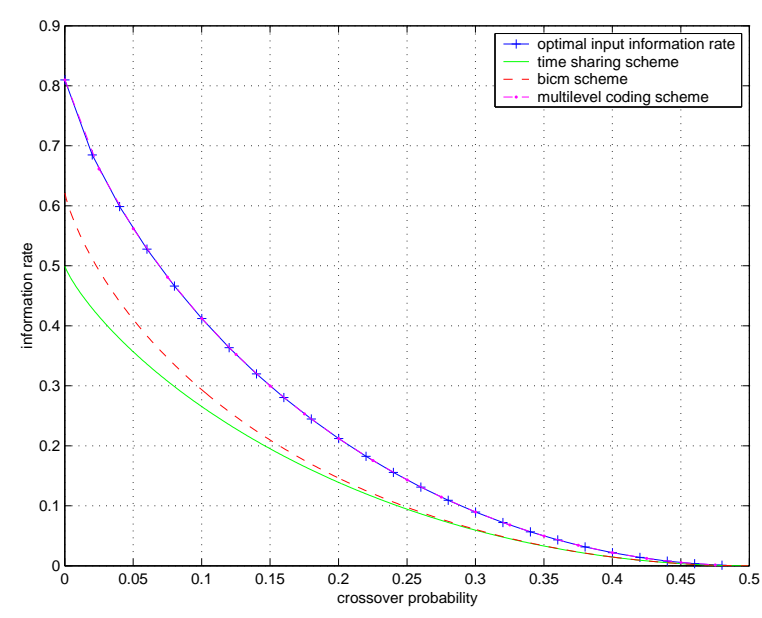

Fig. 3. Achievable Rate of Different Schemes over a BSC with $p_{1}=1 / 4$

The input probabilities that can be induced are of the form $k / 2^{m}$, i.e., dyadic fractional numbers. However, any desired input probability $p_{1}$ can be approached by properly time sharing between two MLC schemes. For instance, if we need $p_{1}=2 / 5$ at the channel input, we can time share between two MLC schemes with 2 layers, one with $p_{1}=1 / 4$ and the other with $p_{1}=1 / 2$. The rate loss of the proposed MLC time sharing scheme is usually small.

Example 2: Consider the proposed MLC over for a BSC with NUI in Figure 4 In this case, the channel crossover probability is fixed $h=0.3$ and the plot shows the change of the information rate as a function of $p_{1}$. We can see that the simple scheme that time shares a linear code with 0s will incur a substantial loss. To get close to the information rate, we may time share between two of the MLC schemes with $m=3$. As shown in Figure 4 for any $k / 8 \leq p_{1} \leq(k+1) / 8$, time sharing between the MLC scheme with $p_{1}=k / 8$ and $p_{1}=(k+1) / 8$ achieves most of the information rate.

\section{RATElEsS TRANSMisSiON SCHEME OVER BINARY} SYMMETRIC CHANNEL USING LAYERING AND REPEATING

In this section, we present an application of the proposed scheme to rateless transmission over the BSC, which is based on layering, interleaving and repeating. We show that in the low rate region, repeating preserves information rate and therefore it can be used as a simple rateless scheme, which extends the result of [7] to the BSC case. We then show that layering information does not incur a rate loss for the BSC due to its degraded nature. As a result, in order to form layering, the coded bits in each layer have to be non-uniformly distributed, where the proposed MLC scheme in Section $\square$ becomes useful.

The problem of rateless transmission over BSC can be formulated as follows: suppose we want to communicate over a BSC with an unknown but lower bounded crossover probability $h \geq h_{\min }$. The capacity of this channel is bounded by $0 \leq C \leq 1-H\left(h_{\min }\right)$. Since $h$ is unknown, the transmitter will first send a mother code of rate $R_{\max }=1-H\left(h_{\min }\right)$ and

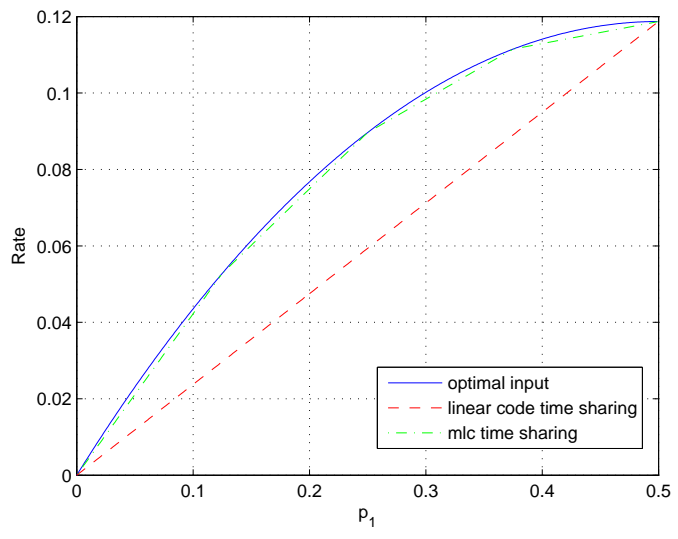

Fig. 4. Information Rate of a 3-Layer MLC Scheme over a BSC with $\mathrm{h}=$ 0.3

then keep sending extra redundancies until the receiver gets enough information to decode. The mother code together with any of the redundant suffix should be a good code such that as long as right enough redundancies are collected, the receiver will be able to decode. Therefore, the proposed scheme is called rateless, since it can work for a wide range of rates $\left(0 \leq R \leq 1-H\left(h_{\min }\right)\right)$. For details on rateless codes over the binary erasure channel (BEC), we refer interested readers to [10][11].

Here, we propose a rateless transmission scheme over the BSC based on layering, interleaving and repeating. The structure of the rateless scheme over the BSC is shown in Figure 5

Proposed Scheme: Encoding: In Block 1, each of the $i^{\text {th }}$ layer encodes its message into coded bits obeying $\left\{p_{i}, 1-p_{i}\right\}$ Bernoulli distribution. The initial code rate of the $i^{t h}$ layer $R_{i}$ should be selected such that $R_{\max }=\sum_{i=1}^{n} R_{i}=1-$ $H\left(h_{\text {min }}\right)$. Then, the coded bits from each layer are interleaved, since we have the NUI distribution, interleaving rather than dithering has to be used to make sure that interference from other layers does not combine coherently when we combine the repeated blocks (See [7] for details). All the interleaved layers are then stacked, i.e., bit wise XORed together $X_{\text {all }}=$ $\sum_{i=1}^{n} \oplus X_{i}$ and transmitted through the BSC. If the receiver is not able to decode, Block 2 is sent. That is all the coded bits of each layer are repeated, interleaved using a different set of interleavers, then stacked together and transmitted through the channel again. The above procedure continues until the receiver has got enough repeated blocks to decode, i.e., $m I\left(X_{\text {all }} ; Y_{\text {all }}\right) \geq R_{\max }$. Decoding: At the decoder, we first wait until enough number of blocks are collected. Then we apply MSD. In the $n^{\text {th }}$ layer, we first generate the soft information of each coded bit from the repeated channel outputs and use them to decode the $n^{t h}$ layer's codeword. Then the decoded bits are subtracted and the $(n-1)^{t h}$ layer sees a clearer channel. We repeat the above decoding procedure until the $1^{\text {st }}$ layer is decoded. Eventually, the information rate after $m$-time repetition is $R=R_{\max } / m$. 


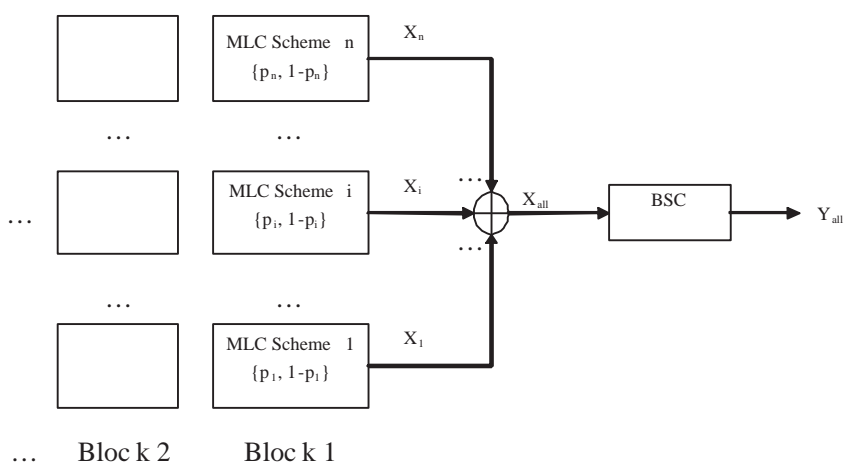

Fig. 5. the Proposed Rateless Scheme over the BSC

As a prerequisite to show the optimality of the proposed scheme, we first give the following lemma:

Lemma 1 Suppose $a$ and $b$ are constants, the function $f(x)=$ $\log (b+a x)$ satisfies the following inequalities as $x \rightarrow 0$

$$
\log b+\frac{a}{b} x-\frac{1}{2}\left(\frac{a}{b} x\right)^{2} \leq \log (b+a x) \leq \log b+\frac{a}{b} x
$$

Proof: This lemma is immediate by considering the Taylor series expansion of $f(x)$ near $x=0$.

It is shown in [12] that for channels with uniform input distribution, repeating preserves information rate, in the low rate region. Here we extend this result to the BSC with NUI.

Theorem 3 Let $X$ be the channel input satisfying Bernoulli distribution $\{p, 1-p\} . X$ is transmitted through a BSC with crossover probability $h$. Let $Y$ be the channel output and $Y^{m}$ be the m-time repetition of $X$ through the BSC. When $p \rightarrow 0$, the information rate is preserved by repeating $X \mathrm{~m}$ times, i.e., $\lim _{p \rightarrow 0} I\left(X ; Y^{m}\right)=\lim _{p \rightarrow 0} m I(X ; Y)$.

Proof: The channel output $Y$ will obey $\left\{p_{y}, 1-p_{y}\right\}$ Bernoulli distribution, where $p_{y}=p \otimes h=p(1-h)+(1-p) h$.

We have the information rate:

$$
I(X ; Y)=H(p \otimes h)-H(h)
$$

As $\mathrm{p}$ goes to zero, we have:

$$
\lim _{p \rightarrow 0} m I(X ; Y)=\lim _{p \rightarrow 0} m p(1-2 h) \log \frac{1-h}{h}+o(p)
$$

On the other hand, we can derive the information combining of a repetition code over the BSC with NUI. Note that $Y^{m}$ can be viewed as a vector channel output, there are $(m+1)$ types of channel outputs. Different outputs of the same type are just different permutations and are statistically equivalent. We have the probability of the $i^{t h}$ type as:

$$
\begin{aligned}
p_{i} & =p h^{i}(1-h)^{m-i}+(1-p) h^{m-i}(1-h)^{i} \\
& =p\left[h^{i}(1-h)^{m-i}-h^{m-i}(1-h)^{i}\right]+h^{m-i}(1-h)^{i}
\end{aligned}
$$

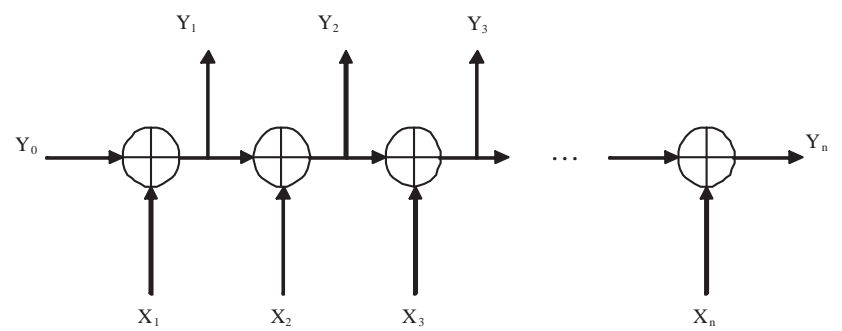

Fig. 6. Degraded Nature of BSC

Let $A_{i}=h^{i}(1-h)^{m-i}-h^{m-i}(1-h)^{i}$ and $B_{i}=h^{m-i}(1-$ $h)^{i}$. Thus we have $p_{i}=A_{i} p+B_{i}$. Since all the probabilities of all the channel outputs will sum up to be " 1 ". We have:

$$
\sum_{i=0}^{m}\left(\begin{array}{c}
m \\
i
\end{array}\right)\left(A_{i} p+B_{i}\right)=1
$$

Besides, since

$$
\sum_{i=0}^{m}\left(\begin{array}{c}
m \\
i
\end{array}\right) B_{i}=(h+1-h)^{m}=1
$$

we have:

$$
\sum_{i=0}^{m}\left(\begin{array}{c}
m \\
i
\end{array}\right) A_{i}=0
$$

The mutual information in the low rate region can therefore be written as:

$$
\begin{aligned}
\lim _{p \rightarrow 0} I\left(X ; Y^{m}\right) & =\lim _{p \rightarrow 0} \sum_{i=0}^{m}-\left(\begin{array}{c}
m \\
i
\end{array}\right) A_{i}\left(\log B_{i}+\log e\right) p+o(p) \\
& =\lim _{p \rightarrow 0} p \log \frac{1-h}{h}\left[-\sum_{i=0}^{m}\left(\begin{array}{c}
m \\
i
\end{array}\right) A_{i} i\right]+o(p) \\
& =\lim _{p \rightarrow 0} p m(1-2 h) \log \frac{1-h}{h}+o(p) \\
& =\lim _{p \rightarrow 0} m I(X ; Y)+o(p)
\end{aligned}
$$

where (15) to 16) follows from Lemma 1 and some straightforward manipulations. From (18, we can see that for very low rate, repeating preserves information rate.

In the proposed scheme, we use layering to drive the coding rate of each layer to the low rate region. We can show that layering is lossless as follows:

Theorem 4 For the BSC, layering does not incur any loss in information rate and MSD can be used to achieve the information rate.

Proof: The channel model of information layering over the BSC is shown in Figure 5 Let the overall stacked 
information as $X_{a l l}=\sum_{i=1}^{n} \oplus X_{i}$ The overall channel output is $Y_{\text {all }}=Y_{n}$. We have the following relationship:

$$
\begin{aligned}
& I\left(X_{1}, X_{2}, \cdots, X_{n} ; Y_{n}\right) \\
& =\sum_{i=1}^{n} I\left(X_{i} ; Y_{n} \mid X_{i+1}, \cdots, X_{n}\right) \\
& =\sum_{i=1}^{n} I\left(X_{i} ; Y_{i}\right)=\sum_{i=1}^{n}\left(H\left(Y_{i}\right)-H\left(Y_{i-1}\right)\right) \\
& =H\left(Y_{n}\right)-H\left(Y_{0}\right)=I\left(X_{\text {all }} ; Y_{n}\right)
\end{aligned}
$$

From the above equations, (21) suggests that stacking does not incur a rate loss and (19) suggests that the overall information rate can be achieved by MSD.

It remains to show that the rate loss due to repeating does not accumulate as the number of layers increases. Thus, as the number of layers goes to be large, the overall rate loss due to repeating is negligible.

Theorem 5 Using layering and repeating for rateless transmission is information lossless as long as the rate of each layer is sufficiently small.

Proof: Let the total number of layers be $N$. For the $j^{\text {th }}$ layer, we have the channel input and output as $X_{j}, Y_{j}$. The input probability $p_{j}$ and crossover probability $h_{j}$. Let the probability of 1 of the overall information be $p_{N}$. For simplicity, let each layer has $p_{i}=p$. By recursion we have the following relationship:

$$
p_{j}=p=\frac{1-\left(1-2 p_{N}\right)^{\frac{1}{N}}}{2}
$$

(Note that $p_{N}=1 / 2-\epsilon$, where $0<\epsilon<1 / 2$, since $p_{i}<1 / 2$. As $N \rightarrow \infty, p_{N}$ can be made arbitrarily close to $1 / 2$.)

The information rate per m-time channel use is $m I\left(X_{j} ; Y_{j}\right)$, while the information rate of the m-time repeating is $I\left(X_{j} ; Y_{j}^{m}\right)$. We have the overall rate loss as:

$\Delta=\lim _{N \rightarrow \infty} \sum_{j=1}^{N} \Delta_{j}=\lim _{N \rightarrow \infty} \sum_{j=1}^{N}\left[m I\left(X_{j} ; Y_{j}\right)-I\left(X_{j} ; Y_{j}^{m}\right)\right]=0$

Note that the information rate of each layer can be made arbitrarily small such that Lemma 1 holds. Thus the information rate per m-time channel use is upper bounded by:

$$
\lim _{p \rightarrow 0} m I\left(X_{j} ; Y_{j}\right) \leq \lim _{p \rightarrow 0} m p\left(1-2 h_{j}\right) \log \frac{1-h_{j}}{h_{j}}
$$

On the other hand, following Lemma 1 we have the following inequalities:

$$
\begin{aligned}
\log \left(B_{i}+A_{i} p\right) & \geq \log B_{i}+\frac{A_{i}}{B_{i}} p-\frac{1}{2}\left(\frac{A_{i}}{B_{i}}\right)^{2} p^{2} \\
\log \left(1-B_{i}-A_{i} p\right) & \geq \log \left(1-B_{i}\right)-\frac{A_{i}}{1-B_{i}} p-\frac{1}{2}\left(\frac{A_{i}}{1-B_{i}}\right)^{2} p^{2}
\end{aligned}
$$

Note that $A_{i}$ and $B_{i}$ do not depend on the number of layers $N$ and consequently they are bounded. Thus, plugging 25
(26) into (15), we have a lower bound of the information rate of the m-time repetition $I\left(X_{i} ; Y_{i}^{m}\right)$ as:

$$
\lim _{p \rightarrow 0} I\left(X_{j} ; Y_{j}^{m}\right) \geq \lim _{p \rightarrow 0} m p\left(1-2 h_{j}\right) \log \frac{1-h_{j}}{h_{j}}-c m p^{2}
$$

where $c$ is constant.

Combining (24) and (27) the rate loss can be bounded by:

$$
\Delta_{j}=m I\left(X_{j} ; Y_{j}\right)-I\left(X_{j} ; Y_{j}^{m}\right) \leq c m p^{2}
$$

Consequently, we have the overall information loss as:

$$
\begin{aligned}
\Delta & =\lim _{N \rightarrow \infty} \sum_{j=1}^{N} \Delta_{j} \\
& \leq c m \lim _{N \rightarrow \infty}\left[\frac{1-\left(1-2 p_{N}\right)^{\frac{1}{N}}}{2}\right]^{2} N \propto \lim _{N \rightarrow \infty} \frac{1}{N}=0
\end{aligned}
$$

Note that $p_{i}<1 / 2$ for all the layers, i.e., each layer will have to have NUI distribution, since otherwise the interference seen by the upper layers will have crossover probability $1 / 2$. Thus, in order to perform the rateless transmission, we need codes for channels with NUI, where the proposed MLC scheme discussed in the previous section becomes useful.

\section{REFERENCES}

[1] R.G. Gallager. Low-Density Parity-Check Codes. MIT Press, Cambridge, MA, 1963.

[2] T. Richardson, M.A. Shokrohalli, and R. Urbanke. Design of capacity approaching irregular low density parity check codes. IEEE Trans. Inform. Theory, 47(2):619-637, Feb. 2001.

[3] T. Richardson and R. Urbanke. Modern Coding Theory. to be published.

[4] R. J. McEliece. Are turbo-like codes effective on non-standard channels? IEEE Information Theory Newsletters, Dec. 2001. based on 2001 ISIT Plenary Lecture.

[5] E. A. Ratzer and D. J. C. Mackay. Sparse low-density parity-check codes for channels with cross-talk. In Proc. ITW, Paris, IL, Oct. 2003.

[6] E. A. Ratzer. Sparse data blocks and multi-user channels. Technical report, University of Cambridge, June 2002.

[7] U. Erez, G. W. Wornell, and M. D. Trott. Faster-than-Nyquist coding: The merits of a regime change. In Proc. Allerton, Monticello, IL, Oct. 2004.

[8] R. G. Gallager. Information Theory and Reliable Communication. New York: Wiley, 1968.

[9] U. Wachsmann, R. F. H. Fischer, and J. B. Huber. Multilevel codes: Theoretical concepts and practical design rules. IEEE Trans. Information Theory, 45(7):1361-1391, July 1999.

[10] M.G. Luby, M. Mitzenmacher, M.A. Shokrollahi, and D.A. Spielman. Efficient erasure correcting codes. IEEE Trans. Inform. Theory, 47(2):569-584, Feb. 2001.

[11] A. Shokrollahi. Raptor codes. Preprint, 2002.

[12] N. Shulman. Universal Channel Coding. PhD thesis, Tel-Aviv University, 2004. 